\title{
Factors associated with duration of breastfeeding
}

\author{
Fatores associados com a duração do aleitamento materno
}

\author{
Roberto G. Chaves ${ }^{1}$, Joel A. Lamounier², Cibele C. César ${ }^{3}$
}

\section{Resumo}

Objetivos: Determinar os índices de aleitamento materno exclusivo e complementado e identificar variáveis que interferem na prática da amamentação no município de Itaúna (MG).

Métodos: Estudo longitudinal realizado com 246 mulheres assistidas na maternidade do Hospital Manoel Gonçalves, no município de Itaúna (MG). O acompanhamento das mães e recém-nascidos foi realizado mensalmente nos primeiros 12 meses após o parto ou até a interrupção da amamentação. A análise da duração do aleitamento materno exclusivo e complementado foi realizada utilizando procedimentos de análise de sobrevivência. O efeito das co-variáveis sobre o tempo de aleitamento foi avaliado através do modelo de regressão de Cox.

Resultados: A prevalência de aleitamento materno exclusivo no sexto mês foi de $5,3 \%$, e de aleitamento materno aos 12 meses, 33,7\%. A mediana de aleitamento materno exclusivo foi de 40 dias, e a mediana de aleitamento materno, 237 dias. A análise multivariada mostrou associação negativa $(p<0,05)$ entre o tempo de aleitamento materno exclusivo e as variáveis: intenção de amamentar ( $<12$ meses), peso do recém-nascido $(<2.500 \mathrm{~g})$ e uso de chupeta. O menor tempo de aleitamento materno foi associado $(p<0,05)$ com idade materna $(<20$ anos), número de consultas de pré-natal ( $<5$ e $>9$ consultas), uso de álcool ou tabaco, tempo da primeira mamada ( $>6$ horas) e uso de chupeta.

Conclusões: Os índices de aleitamento materno no município de Itaúna (MG) estão muito abaixo daqueles preconizados pela Organização Mundial da Saúde. As principais variáveis relacionadas negativamente ao tempo de aleitamento materno exclusivo e complementado estão associadas à assistência materno-infantil, sendo, portanto, passíveis de intervenção.

J Pediatr (Rio J). 2007;83(3):241-246: Aleitamento materno, lactação, desmame.

\begin{abstract}
Objectives: To determine rates of exclusive breastfeeding and of complementary feeding and to identify variables that interfere with breastfeeding in the municipality of Itaúna, MG, Brazil.

Methods: A longitudinal study was undertaken enrolling 246 women who gave birth at the maternity unit of the Manoel Gonçalves Hospital, in Itaúna, MG. The mothers and their infants were seen monthly for the first 12 months after birth or until they stopped breastfeeding. Survival analysis procedures were used to study the duration of exclusive breastfeeding and of complementary feeding. The impact on breastfeeding duration of a series of co-variables was assessed by means of Cox regression modeling.
\end{abstract}

Results: The prevalence of exclusive breastfeeding at 6 months was $5.3 \%$, and for breastfeeding at 12 months it was $33.7 \%$. The median duration of exclusive breastfeeding was 40 days, and median breastfeeding duration was 237 days. Multivariate analysis demonstrated a negative association $(p<0.05)$ between duration of exclusive breastfeeding and the following variables: intended breastfeeding duration $(<12$ months), birth weight of child $(<2,500 \mathrm{~g})$ and use of a pacifier. Shorter breastfeeding duration was associated $(p<0.05)$ with maternal age ( $<20$ years), number of prenatal consultations $(<5$ and $>9$ consultations), use of alcohol or tobacco, delay before first feed $(>6$ hours) and use of a pacifier.

Conclusions: Breastfeeding rates in Itaúna (MG) are well below those recommended by the World Health Organization. The principal variables with a negative relationship with duration of exclusive breastfeeding and of complementary feeding are related to mother and baby health care and, therefore, interventions are possible.

J Pediatr (Rio J). 2007;83(3):241-246: Breastfeeding, lactation, weaning

1. Mestre, Universidade Federal de Minas Gerais (UFMG), Belo Horizonte, MG. Professor auxiliar, Universidade de Itaúna, Itaúna, MG.

2. PhD, University of California (UCLA), Los Angeles, CA, USA. Professor titular, Faculdade de Medicina, UFMG, Belo Horizonte, MG.

3. PhD, UFMG, Belo Horizonte, MG. Professora adjunta, Instituto de Ciências Exatas, UFMG, Belo Horizonte, MG.

Artigo submetido em 05.07.06, aceito em 13.12.06

Como citar este artigo: Chaves RG, Lamounier JA, César CC. Factors associated with duration of breastfeeding. J Pediatr (Rio J). 2007;83(3):241-246.

doi 10.2223/JPED.1610 


\section{Introdução}

A amamentação é uma prática milenar com reconhecidos benefícios nutricionais, imunológicos, cognitivos, econômicos e sociais. Tais benefícios são aproveitados em sua plenitude quando a amamentação é praticada por pelo menos 2 anos, sendo oferecida como forma exclusiva de alimentação do lactente até o sexto mês de vida ${ }^{1}$. Estudos nacionais mostram que, apesar da tendência de melhoria, os índices de aleitamento materno no Brasil estão muito abaixo dos considerados ideais pela Organização Mundial da Saúde $(\mathrm{OMS})^{2,3}$.

O estudo de variáveis demográficas, socioeconômicas, associadas à assistência à saúde e aos hábitos materno-infantis de uma população pode ser de grande utilidade para o conhecimento dos fatores relacionados ao tempo do aleitamento materno exclusivo ou complementado. Assim, podem ser importantes ferramentas no intuito de elevar os índices de aleitamento materno em nosso país. Entretanto, diferenças regionais na prática da amamentação reforçam a necessidade de diagnósticos focais que direcionem a tomada de medidas de intervenção visando apoiar, promover e proteger o aleitamento materno.

O presente estudo teve como objetivo determinar os índices de aleitamento materno exclusivo e complementado e identificar possíveis variáveis que interferem na prática da amamentação no município de Itaúna (MG).

\section{Métodos}

Estudo longitudinal realizado com mulheres assistidas na maternidade do Hospital Manoel Gonçalves de Souza Moreira, único hospital do município de Itaúna, localizado a 72 km de Belo Horizonte, na região metalúrgica do Centro-Oeste de Minas Gerais, com aproximadamente 80.000 habitantes. A clientela assistida pelo hospital pertence a diferentes níveis socioeconômicos, com predominância da classe de menor poder aquisitivo. O acompanhamento das mães e recémnascidos foi realizado nos primeiros 12 meses após o parto ou até a interrupção da amamentação, caso esta ocorresse antes.

O presente artigo foi extraído de um trabalho mais extenso que procurou avaliar a influência do uso de medicamentos sobre o tempo de aleitamento materno. Em face da inexistência de parâmetros para proceder ao cálculo da amostra, foram utilizados os seguintes valores: nível de significância $(\alpha)$ de $5 \%$; poder (1- $\beta$ ) de $90 \%$; tempo de recrutamento de 3 meses; tempo de acompanhamento após término do recrutamento de 12 meses, perda de $20 \%$; probabilidade de estar aleitando ao final do estudo de $30 \%$ para o grupo de mães que utilizaram medicamentos e $70 \%$ para aquelas que não utilizaram; e probabilidade de utilizar medicamento de $25 \%$. Com base nesses parâmetros, o tamanho da amostra calculado foi de 252 mães.
A amostra foi selecionada no período de 01/06/03 a 04/09/03 e incluiu mães residentes em Itaúna que tiveram seus bebês na maternidade do hospital. Deste total, houve as seguintes perdas: cinco mães por não terem sido encontradas na ocasião da primeira entrevista após alta hospitalar e uma por óbito do filho com 40 horas de vida. Das 246 mulheres que compuseram a amostra, oito foram censuradas por perda de acompanhamento, sendo cinco por mudança de município, três por não terem sido encontradas, e 83 foram censuradas pelo término do estudo. Portanto, obteve-se informação exata do tempo de aleitamento materno de 155 mulheres.

Na maternidade, a coleta de dados foi realizada pelo pesquisador em conjunto com acadêmicos da Faculdade de Fisioterapia da Universidade de Itaúna. Os acadêmicos receberam treinamento para aplicação dos questionários em entrevistas com 20 mães em um estudo piloto. Como controle de qualidade, foram realizadas reuniões semanais nas primeiras 8 semanas de coletas de dados e quinzenais até o final do estudo.

Os dados de interesse foram obtidos através de prontuários médicos e entrevistas realizadas no pós-parto imediato. Esses dados forneceram informações sobre características demográficas, socioeconômicas, associadas à assistência de serviços de saúde e aos hábitos materno-infantis. Após a alta da maternidade, o seguimento das mães foi realizado através de contato telefônico ou visita domiciliar.

As variáveis incluídas no estudo foram as seguintes: procedência; plano de saúde; idade materna; cor; estado civil; cirurgia de mama; número de gestações e partos; idade gestacional; pré-natal; renda e consumo de energia familiar; saneamento básico; escolaridade materna e paterna; trabalho materno; licença maternidade; apoio familiar; conhecimento sobre técnicas de amamentação; intenção de amamentar; tempo transcorrido entre o parto e a primeira mamada; uso de medicamentos, álcool e tabaco pela mãe; uso de chupeta; intercorrências; sexo e peso do recém-nascido.

Neste estudo, foram utilizadas as seguintes categorias de aleitamento materno preconizadas pela $\mathrm{OMS}^{1}$ :

- aleitamento materno exclusivo: a criança recebe apenas leite humano de sua mãe ou ama-de-leite, ou leite humano ordenhado, sem outros líquidos ou sólidos, excetuando gotas, xaropes, suplementos minerais ou medicamentos;

- aleitamento materno predominante: a criança recebe leite humano e líquidos, como água, chás, suco de frutas e medicamentos, porém nenhum outro leite;

- aleitamento materno exclusivo mais aleitamento materno predominante (full breastfeeding): não há tradução de consenso na língua portuguesa para o referido termo em inglês; 
- aleitamento materno complementado: a criança recebe leite humano e outros alimentos sólidos ou semi-sólidos;

- aleitamento materno: a criança recebe leite humano (diretamente da mama ou ordenhado) independente de estar recebendo outros alimentos.

O termo desmame total foi atribuído à parada total da amamentação ${ }^{4}$.

O trabalho foi aprovado pelo comitê de ética do Hospital Manoel Gonçalves de Souza Moreira, Departamento Pediatria da UFMG e comitê de ética em pesquisa da UFMG.

A análise estatística dos dados coletados foi realizada pelo programa SPSS ${ }^{5}$, versão 9.0. A análise da duração do aleitamento materno exclusivo e do aleitamento materno foi realizada utilizando procedimentos de análise de sobrevivência. As curvas de aleitamento foram descritas utilizando método de Kaplan-Mayer, que forneceu a mediana do tempo de aleitamento materno exclusivo e de aleitamento materno. A comparação das curvas foi feita através do teste de log rank. O efeito das co-variáveis sobre o tempo de aleitamento foi avaliado através do modelo de regressão de Cox. As variáveis que, na análise bivariada, apresentaram correlação com a variável resposta com valor $p<0,25$ foram incluídas no modelo de regressão multivariada. Foi considerado nível de significância de 5\%.

\section{Resultados}

\section{Duração do aleitamento materno exclusivo e do aleitamento materno}

No momento da alta hospitalar, todas as mulheres amamentavam seus filhos, porém somente 241 (98\%) de forma exclusiva. Em relação ao aleitamento materno exclusivo no primeiro, quarto e sexto meses de vida, as prevalências foram, respectivamente, 62,6, 19,5 e 5,3\%. Para aleitamento materno exclusivo mais predominante no mesmo período, as prevalências foram 77,2, 42,3 e 15\%. Já as prevalências do aleitamento materno no primeiro, quarto, sexto e décimo segundo meses foram de $93,5,75,2$ 58,9 e 33,7\%, respectivamente (Figura 1).

A mediana de aleitamento materno exclusivo foi de 40 dias, e de aleitamento materno exclusivo mais predominante igual a 95 dias. Já a mediana de aleitamento materno foi de 237 dias.

\section{Fatores relacionados ao aleitamento materno exclusivo}

As variáveis que apresentaram associação positiva, com significância estatística pela análise bivariada, com menor tempo de duração do aleitamento materno exclusivo foram peso do recém-nascido $<2.500 \mathrm{~g}(\mathrm{p}=0,03)$, resposta incorreta sobre a técnica do aleitamento $(p=0,012)$, intenção de amamentar por menos de 2 anos $(p=0,009)$, uso de álcool ou tabaco $(p=0,036)$ e uso de chupeta $(p=0,002)$.
Conforme apresentado na Tabela 1, após a análise multivariada, as variáveis associadas negativamente com o tempo de aleitamento materno exclusivo, com significância estatística $(p<0,05)$, foram intenção de amamentar por período menor que 2 anos ( $p=0,002)$, peso do recém-nascido $<2.500 \mathrm{~g}(\mathrm{p}=0,019)$ e uso de chupeta $(\mathrm{p}=0,007)$.

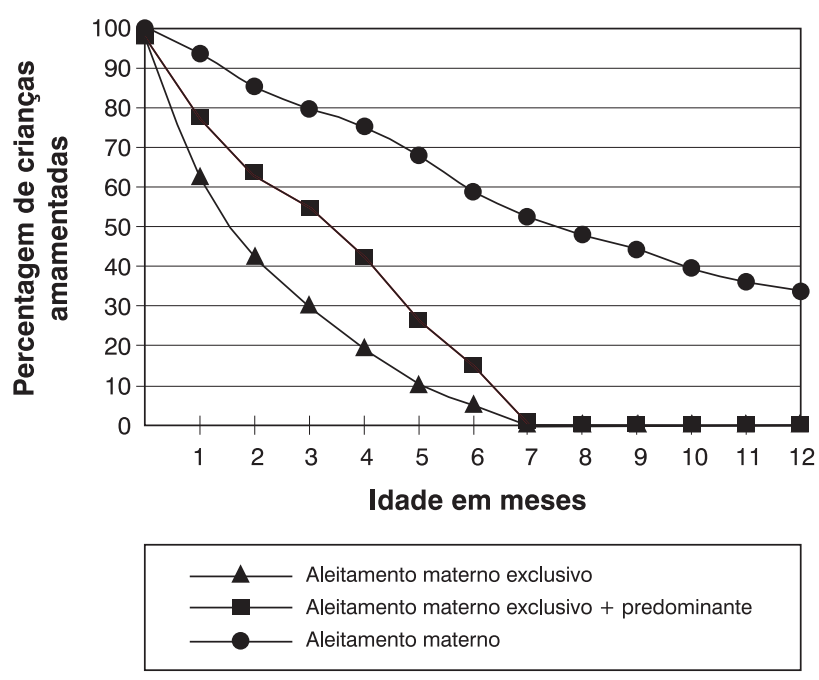

Figura 1 - Prevalência de aleitamento materno exclusivo, aleitamento materno exclusivo mais predominante e aleitamento materno em Itaúna (MG), 2003

\section{Fatores relacionados ao aleitamento materno}

Pela análise bivariada, as variáveis que apresentaram associação negativa com a duração do aleitamento materno foram idade materna menor 20 anos ( $p=0,001)$, idade gestacional $<37$ semanas $(p=0,039)$, número de consultas pré-natal inferior a cinco e superior a nove $(p=0,002)$, primeira mamada após 6 horas de vida $(p=0,032)$, intercorrência com o recém-nascido $(p=0,001)$, uso de álcool ou tabaco $(p=0,001)$ e uso de chupeta $(p=0,000)$.

A análise multivariada demonstrou associação positiva, estatisticamente significativa, entre menor tempo de aleitamento materno e as seguintes variáveis: idade materna menor que 20 anos ( $p=0,009)$, número de consultas pré-natais inferior a cinco ou superior a nove $(p=0,001)$, uso de álcool ou tabaco $(p=0,001)$, primeira mamada após 6 horas de vida $(p=0,038)$ e uso de chupeta $(p=0,000)$. Esses resultados estão descritos na Tabela 2.

\section{Discussão}

Os achados deste estudo foram comparados com dados da literatura, especialmente com aqueles obtidos de estudos populacionais realizados em cidades geográfica e demograficamente semelhantes ao município de Itaúna, embora existam diferenças metodológicas e temporais entre os estudos. Por ser o único hospital da cidade, onde se realiza a quase totalidade dos partos, a amostra pode ser considerada representativa da população. 
Tabela 1 - Variáveis relacionadas ao tempo de aleitamento materno exclusivo $(p<0,05)$ pela análise multivariada, Itaúna (MG), 2003

\begin{tabular}{lll}
\hline Variáveis & RR (IC95\%) & p* \\
\hline & & \\
$\begin{array}{l}\text { Intenção de amamentar } \\
(\geq 24 \text { meses) }\end{array}$ & \\
$\quad<12$ meses & $1,67(1,20-2,33)$ & 0,002 \\
$\quad 12-23$ meses & $1,74(1,23-2,47)$ & 0,002 \\
$\begin{array}{l}\text { Peso do recém-nascido } \\
\text { (<2.500 g) }\end{array}$ & $1,92(1,11-3,33)$ & 0,019 \\
Uso de chupeta & $1,49(1,11-2,00)$ & 0,007 \\
& & \\
\hline & & \\
IC95\% $=$ intervalo de confiança de 95\%; RR = risco relativo. & \\
* Calculadas pelo teste de log rank. &
\end{tabular}

Tabela 2 - Variáveis relacionadas ao tempo de aleitamento materno $(\mathrm{p}<0,05)$ pela análise multivariada, Itaúna (MG), 2003

\begin{tabular}{|c|c|c|}
\hline Variáveis & RR (IC95\%) & p* \\
\hline Idade materna ( $<20$ anos) & $1,89(1,17-3,06)$ & 0,009 \\
\hline \multicolumn{3}{|l|}{$\begin{array}{l}\text { Número de consultas }(\mathrm{n}) \\
\text { no pré-natal }\end{array}$} \\
\hline$<5$ & $0,71(0,34-1,51)$ & 0,379 \\
\hline $5-9$ & $0,33(0,19-0,58)$ & 0,000 \\
\hline $\begin{array}{l}\text { Tempo da primeira } \\
\text { mamada ( }>6 \text { horas) }\end{array}$ & $1,95(1,03-3,67)$ & 0,038 \\
\hline Uso de álcool ou tabaco & $1,92(1,29-2,85)$ & 0,001 \\
\hline Uso de chupeta & $2,99(2,04-4,38)$ & 0,000 \\
\hline
\end{tabular}

IC95\% = intervalo de confiança de 95\%; RR = risco relativo. * Calculado pelo teste de log rank.

Em Itaúna, a prevalência do aleitamento materno exclusivo ao final do sexto mês de vida $(5,3 \%)$ mostrou-se melhor que a prevalência encontrada em Ouro Preto ${ }^{6}(1,8 \%)$, Alto Jequitinhonha ${ }^{7}(0,8 \%)$ e $\mathrm{Embu}^{8}(1,6 \%)$, porém abaixo dos $17,7 \%$ encontrados em Feira de Santana ${ }^{9}$. Já a prevalência de aleitamento materno aos 12 meses foi menor que as encontradas em Ouro Preto ${ }^{6}$ (34,9\%), Montes Claros $741 \%$ ) e Feira de $\operatorname{Santana}^{9}(69,2 \%)$, sendo superior à prevalência de $30 \%$ encontrada em Embu ${ }^{8}$.

A duração mediana de aleitamento materno exclusivo foi de 40 dias, encontrando-se em posição intermediária em relação a outras localidades nacionais. Esse valor é considerado preocupante, pois demonstra a introdução precoce de alimentos, como água, chás e sucos. Esses suplementos são sabidamente desnecessários para hidratação do bebê ${ }^{10}$, elevam o risco de morbidade e mortalidade por infecções ${ }^{11}$, não promovem melhoria no ganho ponderal ${ }^{12}$, além de reduzirem a absorção de ferro e zinco ${ }^{13}$. A introdução precoce desses e de outros alimentos suplementares e também de complementares, como leite não-humano, frutas e papas salgadas, pode resultar na redução da eficácia da amenorréia lactacional como método contraceptivo e também no tempo de amamentação ${ }^{14}$. Além disso, o uso precoce do leite de vaca está associado a risco aumentado para doenças atópicas e diabetes melito tipo $\mathrm{I}^{15}$. A grande freqüência da utilização de suplementos ou complementos alimentares pode, em parte, ser explicada por fatores culturais e pelo desconhecimento por parte de alguns profissionais de saúde dos benefícios do aleitamento materno exclusivo até o sexto mês de vida do lactente.

O valor da mediana de aleitamento materno encontrado em Itaúna foi de 237 dias, sendo superior à mediana de 159 dias em Alfenas ${ }^{16}, 180$ dias em Embu ${ }^{8}, 198$ dias em Ouro Preto ${ }^{6}$ e 205, 9 dias em São José do Rio Preto ${ }^{17}$. Entretanto, manteve-se abaixo da mediana de 261 dias em Montes Claros $^{18} \mathrm{e}$ de 325,5 dias em municípios do Alto Jequitinhonha7. As medianas de aleitamento materno exclusivo e aleitamento materno em Itaúna confirmam a tendência de elevação desses índices em nosso país. Contudo, estão muito abaixo dos valores recomendados pela OMS ${ }^{1}$.

O peso do recém-nascido $<2.500 \mathrm{~g}$, relacionado negativamente com tempo de aleitamento materno exclusivo, também foi descrito em outros estudos ${ }^{7,16,18}$. O fato de recémnascidos de baixo peso serem amamentados exclusivamente por menos tempo que os demais pode ser explicado pelo fato da maior dificuldade que esses bebês apresentam para amamentar, e também pela crença, por parte de alguns profissionais de saúde, que o grande benefício para esses bebês seria o ganho ponderal mais acelerado, lançando mão, para tanto, de fórmulas infantis, farinhas, leite de vaca e açúcar. Portanto, para o aumento dos índices de aleitamento materno exclusivo, é preciso maior atenção para a prevenção de situações que propiciam o nascimento de bebês de baixo peso, como crescimento intra-uterino restrito e parto prematuro, além de medidas como melhoria no atendimento pré-natal e redução do tabagismo durante a gravidez. O método mãecanguru, que propicia maior tempo de contato físico e afetivo entre os recém-nascidos de baixo peso e suas mães, é descrito como ação de impacto positivo sobre a prática da amamentação ${ }^{19}$. Outra ação indispensável seria uma maior atenção dos profissionais de saúde responsáveis pela puericultura no acompanhamento das mães e bebês incluídos nesse grupo, no sentido de incentivar e apoiar o aleitamento materno exclusivo.

O menor tempo de aleitamento materno exclusivo por mães que manifestaram na maternidade intenção de amamentar seus filhos por menos de 2 anos pode ser justificado pelo fato de que essas mulheres estivessem menos informadas sobre a importância do aleitamento materno e mais expostas às pressões externas. Além disso, supõe-se que mães 
com intenção de amamentar por menor período estejam menos empenhadas em seguir as orientações médicas para maior tempo de aleitamento materno exclusivo. Não foi encontrada na literatura relação dessa variável com o tempo de aleitamento materno exclusivo.

O fato de mulheres adolescentes ( $<20$ anos) amamentarem seus filhos por menor tempo que as adultas coincide com os dados publicados por Gigante et al. ${ }^{20}$. Maior tempo de aleitamento materno por mulheres adultas pode ser explicado pelo maior experiência e conhecimento acerca da amamentação por esse grupo de mulheres. Tal fato nos alerta para a necessidade de maior assistência e atenção com as mães adolescentes, no sentido de orientação, incentivo e apoio à amamentação. A divulgação de informações sobre educação sexual poderia contribuir para a redução da freqüência de gravidezes na adolescência.

As mulheres que realizaram menos de cinco e mais que nove consultas de pré-natal amamentaram seus filhos por menos tempo que aquelas que fizeram entre cinco e nove consultas. Caldeira \& Goulart ${ }^{18}$, em Montes Claros, encontraram que as mulheres que realizaram menos de cinco consultas tiveram menor tempo de aleitamento materno que aquelas que realizaram mais de cinco consultas $(p=0,0496)$, porém somente em análise bivariada. $O$ fato de mulheres com menos de cinco consultas no pré-natal amamentarem seus filhos por menos tempo pode estar relacionado ao menor acesso às informações sobre aleitamento fornecidas durante o pré-natal. Contudo, a associação entre maior número de consultas de pré-natal e menor tempo de aleitamento materno não pôde ser explicado de forma satisfatória. Porém, pode-se especular sobre um maior grau de ansiedade e insegurança nessas mulheres. Para melhor explicação desse achado, seria necessário maior estudo desse grupo de pacientes, inclusive utilizando métodos qualitativos de avaliação.

Menor tempo de aleitamento materno por recém-nascidos que mamaram após as primeiras 6 horas de vida também foi descrito por Caldeira \& Goulart ${ }^{18}$, em Montes Claros. Bautista ${ }^{21}$ também descreveu a relação positiva entre início mais rápido da primeira mamada e maior tempo de aleitamento materno. Já Figueiredo \& Goulart ${ }^{22}$ notaram associação positiva entre a redução nos índices de desmame total com aumento do número de mulheres que não foram separadas de seus filhos nas primeiras 6 horas de vida. Esse achado pode ser justificado pelo efeito benéfico desse primeiro contato para a mãe, levando maior liberação de ocitocina, favorável à ejeção do leite, além do efeito lactogênico da sucção do bebê. Além disso, é descrita a importância do contato precoce entre mãe e filho no fortalecimento do vínculo afetivo entre os mesmos, fato que pode ser responsável por maior tempo de aleitamento ${ }^{23}$. O maior tempo de aleitamento materno pelos bebês que iniciaram a amamentação mais precocemente demonstra o importante papel das práticas hospitalares sobre o sucesso da amamentação, reforçando a importância da prática integral do alojamento conjunto, permitindo que mãe e filho permaneçam juntos durante todo o período de permanência hospitalar ${ }^{24}$.

Mulheres que relataram na maternidade fazer uso de álcool e tabaco tiveram menor tempo de aleitamento que aquelas que relataram não fazer uso dessas drogas. Apesar de evidências que amamentação associada ao tabagismo materno é menos prejudicial à criança que o uso de leites industrializados ${ }^{25}$, a maioria dos dados da literatura associa menor tempo de aleitamento materno ao tabagismo materno ${ }^{26}$. Além disso, tanto o tabagismo quanto o uso de álcool têm sido descritos como práticas associadas à redução do volume de leite materno 27,28 . Apesar de a Academia Americana de Pediatria (AAP) ${ }^{29}$ considerar o álcool compatível com a amamentação e permitir o uso do tabaco, os resultados deste estudo reforçam a importância de esforços no sentido de reduzir o uso dessas drogas pelas mulheres, em especial pelas nutrizes. Devido aos fatores físicos e psíquicos envolvidos nos períodos de gravidez e amamentação, esses momentos tornam-se ideais para que os profissionais de saúde orientem a interrupção do uso de álcool e tabaco.

O uso da chupeta pelo lactente foi associado negativamente tanto com tempo de aleitamento materno exclusivo quanto ao aleitamento materno. Nas análises, considerou-se somente a possibilidade de o bebê ter usado ou não a chupeta, não determinando freqüência ou tempo do uso. O maior tempo de aleitamento materno exclusivo por bebês que não fizeram uso de chupeta está de acordo com dados da literatura $^{26,30}$. Esse fato pode estar relacionado à maior segurança das mães dos bebês que não usaram chupeta, estando estas menos sensíveis às pressões sociais e menos ansiosas em relação ao crescimento de seus filhos. Além disso, a chupeta pode estar relacionada à diminuição da produção de leite, em razão da redução da freqüência das mamadas ${ }^{30}$. Silveira \& Lamounier $^{26}$ realizaram revisão de literatura sobre estudos que associaram uso de chupeta com menor duração do aleitamento materno. Todos os oito estudos encontrados apresentaram associação estatisticamente significativa. Soares et al. ${ }^{30}$ encontraram risco 2,8 vezes maior de desmame total até o sexto mês por crianças ainda amamentadas até 1 mês de vida que estavam fazendo uso de chupeta. Portanto, o uso de chupeta pode estar camuflando dificuldades na amamentação ou mesmo ansiedade e insegurança materna frente ao processo alimentar, fatos que nos alertam para a necessidade de solucionar tais problemas. Seria desejável a realização de estudos que avaliem mais profundamente este tema, utilizando inclusive métodos qualitativos de avaliação. Assim, as mães precisam ser melhor informadas sobre as graves conseqüências do uso de chupeta pelo lactente, hábito aparentemente inócuo e muito aceito culturalmente, em relação ao sucesso da amamentação. 


\section{Considerações finais}

A determinação dos indicadores e das variáveis relacionados ao tempo de aleitamento materno, exclusivo ou total, pode ser um valioso instrumento no planejamento de ações e políticas locais no sentido de melhorar os índices de aleitamento materno. Este estudo mostra que, a exemplo dos demais estudos nacionais, os índices de aleitamento materno estão muito abaixo daqueles preconizados pela OMS. Revela ainda que as principais variáveis relacionadas negativamente ao tempo de aleitamento materno exclusivo e complementado são passíveis de intervenção. Assim, a melhoria na qualidade da assistência à saúde materno-infantil pode ser considerada uma medida de extrema importância para o aumento nos índices de aleitamento materno.

\section{Referências}

1. World Health Organization. Report of the expert consultation on the optimal duration of exclusive breastfeeding: conclusions and recommendations. Geneva: WHO; 2001.

2. Brasil, Ministério da Saúde. Prevalência de aleitamento materno nas capitais brasileiras e no Distrito Federal. Brasília: Ministério da Saúde; 2001.

3. Lamounier JA. Tendências do aleitamento materno no Brasil. Rev Med Minas Gerais. 1999;9:59-66.

4. World Health Organization, United Children's Fund. Complementary feeding of young children in developing countries: a review of current scientific knowledge. Geneva: WHO/NUT/98.1; 1998.

5. SPSS for Windows. Release 9.0. Chicago: SPSS; 1998.

6. Passos MC, Lamounier JA, Silva CAM, Freitas SN, Baudson MFR. Práticas de amamentação no município de Ouro Preto, Minas Gerais, Brasil. Rev Saude Publica. 2000;34:617-22.

7. Silveira FJF, Lamounier JA. Prevalência do aleitamento materno e prática de alimentação complementar em crianças com até 24 meses de idade na região do Alto Jequitinhonha, Minas Gerais. Rev Nutr. 2004;17:437-47.

8. Pedroso GC, Puccini RF, Silva EMK, Silva NN, Alves MCGP. Prevalência de aleitamento materno e introdução precoce de suplementos alimentares em área urbana do Sudeste do Brasil, Embu, São Paulo. Rev Bras Saude Matern Infant. 2004;4:45-58.

9. Vieira GO, Almeida JAG, Silva LR, Cabral VA, Netto PVS. Fatores associados ao aleitamento materno e desmame em Feira de Santana, Bahia. Rev Bras Saude Matern Infant. 2004;4:143-50.

10. Brown $\mathrm{KH}$, Creed de Kanashiro $\mathrm{H}$, del Aguila R, Lopez de Romana G, Black RE. Milk consumption and hydration status of exclusively breast-fed infants in a warm climate. J Pediatr. 1986; 108:677-80.

11. Arifeen S, Black RE, Antelman G, Baqui A, Caulfield L, Becker S. Exclusive breastfeeding reduces acute respiratory infection and diarrhea deaths among infants in Dhaka slums. Pediatrics. 2001;108:E67.

12. Cohen RJ, Brown KH, Canahuati J, Rivera LL, Dewey KG. Effects of age of introduction of complementary foods on infant breast milk intake, total energy intake, and growth: a randomised intervention study in Honduras. Lancet. 1994;344:288-93.

13. Black RE, Victora CG. Optimal duration of exclusive breast feeding in low income countries. BMJ. 2002;325:1252-3.

14. Rea MF. Os benefícios da amamentação para a saúde da mulher. J Pediatr (Rio J). 2004;80:S142-6.
15. Monte CMG, Giugliani ERJ. Recomendações para a alimentação da criança amamentada. J Pediatr (Rio J). 2004;80(5 Supl):S131-41.

16. Pereira FA. Aspectos sociodemográficos e médico-hospitalares do aleitamento materno em Alfenas, Minas Gerais [dissertação]. Alfenas: Faculdade de Medicina da UNIFENAS; 2000.

17. Figueiredo MG, Sartorelli DS, Zan TAB, Garcia E, Silva LC, Carvalho FLP, et al. Inquérito de avaliação rápida das práticas de alimentação infantil em São José do Rio Preto/SP, Brasil. Cad Saude Publica. 2004;20:172-9.

18. Caldeira AP, Goulart EM. A situação do aleitamento materno em Montes Claros, Minas Gerais: estudo de uma amostra representativa. J Pediatr (Rio J). 2000;76:65-72.

19. Venâncio SI, de Almeida H. Método mãe canguru: aplicação no Brasil, evidências científicas e impacto sobre o aleitamento materno. J Pediatr (Rio J). 2004;80:S173-80.

20. Gigante DP, Victora CG, Barros FC. Nutrição materna e duração da amamentação em uma coorte de nascimento de Pelotas/RS. Rev Saude Publica. 2000; 34:259-65.

21. Bautista LE. Duration of maternal breast-feeding in the Dominican Republic. Rev Panam Salud Publica. 1997;1:104-11.

22. Figueiredo LM, Goulart EM. Análise da eficácia do Programa de Incentivo ao Aleitamento Materno em um bairro periférico de Belo Horizonte (Brasil): 1980/1986/1992. J Pediatr (Rio J). 1995;71:203-8.

23. Widstrom AM, Wahlberg V, Matthiensen AS, Eneroth $P$, UvnasMoberg K, Werner S, et al. Short-term effects of early suckling and touch of the nipple on maternal behavior. Early Hum Dev. $1990 ; 21: 153-63$

24. Organização Mundial da Saúde. Evidências científicas dos dez passos para o sucesso no aleitamento materno. Brasília: OPAS; 2001.

25. Batstra L, Neeleman J, Hadders-Algra M. Can breast feeding modify the adverse effects of smoking during pregnancy on the child's cognitive development? J Epidemiol Community Health. 2003;57:403-4.

26. Silveira FJF, Lamounier JA. Influência do uso da chupeta e do tabagismo materno na amamentação: revisão de literatura. Rev Med Minas Gerais. 2003;13:120-8.

27. Vio F, Salazar G, Infante C. Smoking during pregnancy and lactation and its effects on breast-milk volume. Am J Clin Nutr. $1991 ; 54: 1011-6$

28. Cobo E. Effect of different doses of ethanol on the milk-ejecting reflex in lactating women. Am J Obstet Gynenol. 1973; 115:817-21

29. American Academy of Pediatrics Committee on Drugs. Transfer of drugs and other chemicals into human milk. Pediatrics. 2001;108:776-89.

30. Soares ME, Giugliani ER, Braun ML, Salgado AC, de Oliveira AP, de Aguiar PR. Uso de chupetas e sua relação com o desmame precoce em população de crianças nascidas Hospital Amigo da Criança. J Pediatr (Rio J). 2003;79:309-16.

Correspondência:

Roberto Gomes Chaves

Rua Mariângela Medeiros, 50, Centro

CEP 35680-457 - Itaúna, MG

Tel.: (37) 3241.5194

Fax: (37) 3242.1064

E-mail: robertogchaves@uol.com.br 